\title{
Changing paradigms in lower extremity reconstruction in war-related injuries
}

\author{
Margaret Connolly', Zuhaib R. Ibrahim ${ }^{2^{*}}$ and Owen N. Johnson $\|^{3}$
}

\begin{abstract}
Background: Ballistic high-energy trauma has substantially increased the severity of non-fatal extremity injuries incurred in modern warfare. Expedient medical care, refinement in surgical techniques, and soft tissue coverage have brought about a paradigm shift in the management of lower extremity wounds during the last decade with an increased emphasis on limb salvage.

Methods: A literature-based study was conducted to analyze reconstructive modalities based on the location, depth, and severity of wounds, as well as mechanism of injury, concomitant vascular injuries and open fractures, choice of flap, timing of definitive reconstruction, and complications.

Results: Extremity injuries account for over $60 \%$ of injuries in the recent conflicts in Iraq and Afghanistan, with the majority secondary to explosive devices. The severity of these injuries is profound compared with civilian registries, and conventional injury scoring systems have failed to accurately predict outcomes in combat trauma. The mainstay of treatment is serial debridement, negative pressure therapy, fracture stabilization, and treatment of concomitant injuries by the forward medical teams with subsequent definitive reconstruction after transport to an advanced military treatment facility. Autologous reconstruction with free tissue transfer and pedicled flaps remains the primary modality for soft tissue coverage in limb salvage. Adjunct innovative modalities, such as external tissue expansion, dermal substitutes, and regenerative matrices, have also been successfully utilized for limb salvage.
\end{abstract}

Conclusion: Lower extremity injuries account for the vast majority of injuries in modern warzones. Explosive devices represent the most common mechanism of injury, with blast impact leading to extensive soft tissue injuries necessitating complex reconstructive strategies. Serial debridement, negative pressure therapy, and autologous reconstruction with free tissue transfer and pedicled flaps remain the mainstay of treatment in recent conflicts.

Keywords: Limb salvage, Free flap, Pedicled flap, Lower extremity, Blast injury, Combat, Battlefield, Reconstruction

\section{Background}

Lower extremity reconstruction following modern battlefield trauma poses significant challenges for the reconstructive surgeon compared with lower extremity injuries seen in the civilian population $[1,2]$. High-energy blast impact from improvised explosive devices (IEDs) leads to an extensive zone of injury, which often results in limited donor site options for reconstruction [3, 4]. Inadequate perfusion, heavily contaminated open fractures, exposed bone with stripped periosteum, and devitalized tendons add to the complexity of these cases [5, 6]. Although amputation is inevitable, and even the preferred treatment

\footnotetext{
*Correspondence: zibrahi2@jhmi.edu

${ }^{2}$ Department of Plastic and Reconstructive Surgery, Johns Hopkins University

School of Medicine, 601 N Caroline St., Baltimore, MD 21287, USA

Full list of author information is available at the end of the article
}

in certain cases [7], successful limb salvage with optimal functional recovery can make a remarkable difference in the quality of life for this relatively young and otherwise healthy population. With technological advancements, limb salvage has been reported in as high as $93 \%$ of patients from recent conflicts with high-energy ballistic injuries $[1,8]$.

Early return to ambulation, complete bone healing, and durable soft tissue coverage are key goals of successful limb salvage in complex lower extremity trauma $[7,9]$. In this context, the concept of the reconstructive ladder exists as a guide for choosing the appropriate method of reconstruction in civilian injuries [10]. However, highly encouraging reconstructive results from innovative modalities, such as dermal substitutes, regenerative matrices, pedicled 
perforator flaps, and tissue expansion in the civilian population have made the decision making more complex and individualized [10, 11]. Despite abundant literature demonstrating successful soft tissue coverage using these advanced technologies in the civilian population, the utility of such modalities in the military setting has not been thoroughly examined. Additionally, contemporary reconstructive strategies may not be applicable to the extensive injuries from blast impacts in battlefield trauma.

\section{Review}

\section{Methods}

A literature-based study was conducted using PubMed, Cochrane, and Embase databases for articles published in English ranging from 2005 to 2015. This included the following search terms: "lower extremity," "reconstruction," "flap," "military," "combat," and "battlefield." For historical and technical background, articles published prior to 2005 were also reviewed if they described a historical perspective of currently utilized modalities. For the purpose of this review, articles limited to amputation or vascular repair without pertinent data on soft tissue reconstruction were excluded. Similarly, single patient case reports were excluded unless they described a significant adverse outcome related to a reconstruction modality.

\section{Results}

The majority of contemporary scientific evidence for advanced treatment of combat-related lower extremity trauma comes from the current conflicts in Iraq and Afghanistan. The data sources for these studies include the Department of Defense Trauma Registry, US Navy and US Marine Corps Combat Trauma Registry Expeditionary Medical Encounter Database, and hospital-based data from military treatment facilities. In addition, a case-control study for lower extremity reconstruction of landmine-related injuries is included in this review [12]. Available literature for combat-related injuries consists of a retrospective cohort of patients with lower extremity wounds (Evidence Level 3).

\section{Mechanism of injury}

In contrast to civilian injuries, penetrating trauma accounts for the vast majority of lower extremity injuries, with $63.3 \%$ of injuries resulting from explosive devices and $16.3 \%$ from high-velocity gunshot wounds. These explosive devices also result in devastating blunt injuries in the form of blast effect, shockwave, intimal damage, and closed brain injury [13].

\section{Timing of surgery}

Godina [14] demonstrated that failure and complication rates of free-tissue transfer in civilian patients increase if the operation is performed more than $72 \mathrm{~h}$ after injury. However, there are significant differences in combat injuries, which can be contraindications to immediate free tissue transfer. In the senior author's opinion, provision of definitive care in the battlefield poses several challenges and, hence, the US military doctrine is to stabilize and evacuate the wounded soldier with definitive reconstruction performed after transfer. In the recent conflicts, the average time from injury on the battlefield to arrival at a local tertiary medical center was 4 to 10 days [15]. Final disposition is reserved for when the patient is least critically ill and at the facility best-equipped for treatment and reconstruction. Combat wounds are associated with heavy contamination and devitalized tissue, usually requiring serial debridement, proper broad spectrum intravenous antibiotics, and complex fracture management prior to definitive coverage [2,3]. Kumar et al. reported 12 free and 62 pedicled flaps performed in a sub-acute period after serial debridement with complete failure rates of 0 and $1.4 \%$, respectively [2]. In a later series, Kumar et al. reported 46 flaps (10 free and 36 pedicled) for lower extremity reconstruction with only one flap loss [3]. Hence, emergent revascularization, resuscitation, stabilization, fracture reduction, debridement, and negative pressure therapy with definitive flap coverage in a sub-acute period remains the mainstay of treatment.

Because traditional scoring systems such as the Mangled Extremity Severity Score (MESS) fail to accurately predict the outcome of limb salvage in ballistic wounds [7], the decision to perform an amputation versus limb salvage is reserved until evacuation and transport to an advanced treatment facility. Brown et al. [7] reported a retrospective series of 77 military personnel with 86 mangled ballistic lower extremity wounds and assessed the predictability of the MESS system. Traditionally in the civilian population, a MESS of 7 or greater is considered an indication for amputation [16]. However, this criterion resulted in positive predictive value of only $64.3 \%$ in ballistic injuries, bringing its applicability in combat wounds into question [7]. According to this report, prolonged hypotension and ischemia were the strongest predictors of failed limb salvage in ballistic lower extremity injuries [7]. The MESS was also not helpful in predicting which patients referred to a limb salvage clinic would ultimately require late amputation [17].

Microvascular free tissue transfer for host nation patients was also performed within the combat support hospitals with over a $90 \%$ success rate [4] in the sub-acute phase (within 1 month from the initial injury). In various parts of the world with limited resources and equipment, these results are more applicable for conflicts and add to the importance of microvascular training and expertise needed in military medicine, particularly at support hospitals. 


\section{Method of reconstruction}

Autologous tissue transfer with either pedicled or free flaps has remained the pillar of treatment for definitive reconstruction in recent conflicts. Sabino et al. [18] reported 10 years of experiences at Walter Reed National Military Medical Center where 359 flaps were performed for war-related extremity trauma (143 free and 216 pedicled flaps), including poly-extremity trauma and reconstruction, with outcomes comparable to other military and civilian lower extremity reconstruction. In this series, $55 \%$ of flaps were muscle flaps, and $42 \%$ were fasciocutaneous flaps. The most common free muscle flap was the latissimus dorsi (13\%), the most common free fasciocutaneous flap was the anterolateral thigh (11\%), the most common pedicled muscle flap was the gastrocnemius (17\%), and the most common pedicled fasciocutaneous flap was the sural flap (6\%). Historically, a massive zone of injury in ballistic wounds was considered to be a precluding factor for a rotational flap; however, Burns et al. [19] demonstrated that pedicled flaps are a safe option for carefully selected cases of severely injured extremities. A case control study from the Turkish Armed Forces Rehabilitation Center demonstrated no significant differences in functional ambulation scale, energy expenditure, and a $10 \mathrm{~min}$ walking test when comparing free muscle flap coverage of severe landmine-related heel defects to healthy volunteers [12]. This highly encouraging functional outcome advocates for the use of autologous free tissue transfer for foot and ankle defects.

\section{Concomitant vascular injuries and open fractures}

Concomitant vascular injuries are common in ballistic lower extremity wounds. In a recent report, $24 \%$ of patients requiring flap coverage for ballistic lower extremity wounds had concomitant vascular injury that required emergent repair [20]. Flap coverage was performed at an average of 31 days following vascular repair with an overall flap failure and complication rate of 8 and $31 \%$, respectively, which was comparable to the complication rate of flaps not requiring vascular repair (10 and $28 \%$, respectively). However, variability in the flap success rate exists among different series from a variety of centers and time periods, primarily based on the extent of lower extremity trauma, which became progressively worse in later time periods. Treatment strategies and technical skills also improved over time leading to heterogeneity in outcomes.

Dickens et al. [21] analyzed the risk factors for amputation versus limb salvage in open calcaneal fractures (102 open fractures: 43 amputations, 59 limbs salvaged) and reported a progressively increased risk of amputation with increased wound area. Unsuccessful limb salvage was also significantly associated with ipsilateral forefoot fracture, talar fracture, plantar wound, and positive wound culture.
In this series, $15 \%$ of patients had delayed amputation (greater than 12 weeks post-injury). In contrast, in the civilian population, the Lower Extremity Assessment Project (LEAP) study reported a delayed amputation rate of only $3.9 \%$ [22]. Of note, lack of plantar sensation was not significantly associated with increased risk of amputation. Expert opinion on strategies for managing ballistic foot wounds also suggests a high likelihood of amputation in open calcaneal fractures with extensive plantar soft tissue loss [23, 24].

Similarly, Doucet et al. [13] compared open tibial fractures sustained in combat with a civilian registry and reported 21 amputations out of 115 (18 \%) open tibial fractures in combat-related injuries compared with only 45 amputations out of 850 (5\%) civilian open tibial fractures. Although combat-related open tibial fractures are associated with a higher incidence of amputation, limb salvage was possible in almost $82 \%$ of these injuries.

\section{Adjunct reconstructive modalities}

Innovative reconstructive strategies, such as dermal substitutes, regenerative matrices, and tissue expansion, are now commonly utilized in lower extremity reconstruction in civilian trauma [25]. Fleming et al. [26] demonstrated successful use of these modalities in single patient case reports, primarily used as an adjunct to traditional flaps for preserving limb length in war-related amputation stumps. In this context, a biologic composite dermal substitute (Integra Life Sciences Inc.) with staged skin was used to provide a pliable surface for prosthetic use while preserving a functional limb segment. In another retrospective series, 15 out of 16 wounds with significant soft tissue loss were successfully reconstructed with a bilayered dermal substitute and staged skin grafting [27]. Similarly, external tissue expanders were used for delayed primary closure to provide a pliable surface for prosthesis fitting [26].

Valerio et al. [11] reported a case series of 51 patients where a porcine urinary bladder matrix was applied as a biologic scaffold (MatriStem MicroMatrix ${ }^{\bullet}$ ACell, Inc., MD, USA) to promote granulation over either tendon without paratenon or bone without periosteum. Success was seen in $86 \%$ of wounds with MatriStem application that proceeded to full healing with skin graft, dermal substitute, or flap coverage. Although subjective observational findings demonstrated constructive remodeling and a healthy vascularized wound bed, the study had inherent limitations, including lack of a control group and varying numbers of procedures prior to application of the biologic scaffold.

Intraoperative indo-cyanine green laser angiography (ICGLA) has also been used as a guidance tool for complex debridement in heavily contaminated wounds, soft tissue avulsion injuries, amputation stumps, and flap design and assessment [28]. In a retrospective series, intra- 
operative plans were modified due to perfusion related issues in 35 out of 186 (18.8 \%) patients, as detected by ICGLA. These reports provide promising results for future use of innovative modalities in war-related injuries.

\section{Complications}

Flap failure, hematoma, chronic pain, non-union, osteomyelitis, heterotopic ossification, and delayed amputation represent the major complications reported in warrelated extremity reconstructions $[5,18]$. Overall, the success rate for flap coverage exceeds $90 \%[2-4,18]$. Sabino et al. [18] reported flap failure leading to amputation in only four out of 359 cases of flap coverage (1\%). There was no difference in the overall complication rate between muscle and fasciocutaneous flaps. Similarly, Huh et al. [5] reported 213 open (Gustilo-Anderson) [29] Type 3 tibial fractures out of which 177 were managed with limb salvage, while the remainder underwent primary amputation within 12 weeks of injury. Eleven out of 177 patients underwent late amputation with deep soft tissue infection and osteomyelitis as the most significant risk factors for late amputation. These patients with late amputation required a significantly higher number of revision procedures and had a higher incidence of flap failure secondary to deep infection. In a retrospective analysis of 964 US military personnel with major lower extremity injuries, Melcer et al. [30] found early amputees and limb salvage patients had comparable physical complication rates; however, limb salvage patients had significantly higher psychological diagnoses. Late amputees ( $>90$ days) had significantly higher physical and psychological complications than both early amputees and limb salvage patients.

\section{Discussion}

The mechanism of injury, depth, and location of the wound are important considerations when selecting a method of reconstruction for complex lower extremity wounds. However, regardless of the method of reconstruction, adequate debridement of devitalized tissue is critical for overall success of any reconstructive modality, and this is of particular importance for lower extremity wounds where tissue perfusion can be marginal [2]. Thorough debridement and irrigation is essential to removing foreign materials that may be embedded in the tissue secondary to blast injuries [31]. Inadequate debridement can lead to persistent infection, osteomyelitis, and delayed amputation. Such complications may persist despite aggressive and repeated debridement. Although innovative technologies, such as a bi-layered tissue regenerative matrix with staged skin grafts and tissue expanders, have been utilized in lower extremity reconstruction in civilian population [32], their utility as definitive coverage has yet to be determined in war-related injuries. Heavy contamination in combat zones and the presence of multi-level open fractures have precluded the widespread use of these technologies for commonly encountered ballistic injuries in recent conflicts.

War-related injuries are often associated with polyextremity trauma. Multiple free flaps in the same patient for multiple limb salvage have demonstrated no significant difference in overall complication rate. However, the reported free flap failure rate risk in multiple limb salvage cases is significantly higher compared with single limb salvage [33].

With technological advances in resuscitative trauma care and fracture management, reconstructive options have evolved as well, with more injuries salvaged than ever before [7]. This further adds to the importance attempting limb salvage at the initial encounter. However, according to the Military Extremity Trauma Amputation/Limb Salvage (METALS) study [9], those treated with amputation had a better functional outcome than limb salvage at 3 years post-injury, with both groups demonstrating moderate to severe physical and psychosocial disability. Delayed physical and psychological complications may be related to differences in rehabilitation options for amputees and limb salvage patients. While limb salvage clinics exist, patients are typically referred only when the injured limb is at risk of late amputation [17]. In contrast, there are established military amputee care programs (ACPs) more readily available to amputees [30]. Differences in rehabilitation protocols, extent and severity of injury, and potential selection bias together present challenges in drawing a universal conclusion based on this study alone. In this context, elective amputation versus further reconstruction for limb salvage at definitive medical treatment centers plays a pivotal role after a thorough discussion of potential challenges, including prolonged recovery, persistent disability, and need for multiple revision procedures.

Pedicled perforator flaps based on perforasome theory are now commonly utilized for lower extremity reconstruction in the civilian population [34]. Due to extensive soft tissue injury and the potential violation of fascial planes and perforators in ballistic injuries, island flaps based on perforators are uncommon in war-related patients. Traditional pedicled flaps, such as the gastrocnemius muscle and sural fasciocutaneous flaps, however, are the most common methods of reconstruction in reported literature $[3,18]$.

The majority of current evidence is based on reports from US military medical centers where amputees undergo aggressive and state-of-the-art rehabilitation. These patients have access to advanced prosthetic devices, and amputation is a more socially acceptable option within this community $[13,35]$. For the rest of the population, there may be logistic, social, and cultural reasons that lead to different functional and psychosocial outcomes following amputation compared with limb salvage. The mechanism and pattern 
of injuries may also differ based on evacuation systems, healthcare delivery systems, and the availability of advanced body armor. Global data on the outcome of combat-related lower extremity injuries in current conflicts outside of the studies discussed are limited, and the technical considerations on the choice of limb salvage versus amputation, as well as the choice and timing of flap, may depend on available resources and expertise.

\section{Conclusions}

Advancements in body armor, multidisciplinary trauma resuscitation, and surgical care have resulted in improved combat casualty survival rates; however, they have also lead to an increase in non-fatal extremity injuries from explosive devices. Ballistic impact from these injuries often causes an extensive zone of injury with multi-level open fractures and soft tissue defects. Resuscitative care, serial debridement, negative pressure wound therapy, and fracture stabilization remain the mainstay of early treatment, while definitive coverage is performed at military treatment facilities in a sub-acute manner. Reconstructive modalities, such as dermal substitutes, regenerative matrices, and external tissue expansion, have been successfully explored as adjunct modalities in the treatment of war-related injuries. Pedicled and free tissue transfer, however, are consistently the primary methods of definitive soft tissue coverage. Both limb salvage and amputation are associated with moderate to severe functional disability. Short and intermediate term results have shown significantly improved functional results with early amputation when compared with limb salvage in combat trauma. However, the global impact of these results is unknown due to cultural, social, and logistic differences in aggressive rehabilitation and the availability of advanced prosthetic devices.

\section{Abbreviations}

ICGLA: Indo-cyanine green laser angiography; IED: Improvised explosive device; LEAP: Lower Extremity Assessment Project; MESS: Mangled Extremity Severity Score; METALS: Military Extremity Trauma Amputation/Limb Salvage.

\section{Competing interests}

The authors declare that they have no conflicts of interest.

\section{Authors' contributions}

$\mathrm{ZI}$ conducted the literature review and drafted the manuscript. MC contributed to review and edited and formatted the final manuscript. OJ edited and contributed to the revised manuscript. All authors read and approved the final manuscript.

\section{Author details}

'Department of Surgery, University of Maryland Medical Center, 22 S Greene St., Baltimore, MD 21201, USA. ${ }^{2}$ Department of Plastic and Reconstructive Surgery, Johns Hopkins University School of Medicine, 601 N Caroline St.,

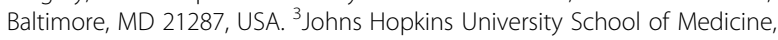
601 N Caroline St., Baltimore, MD 21287, USA.

Received: 15 September 2015 Accepted: 21 March 2016 Published online: 31 March 2016

\section{References}

1. Geiger S, McCormick F, Chou R, Wandel AG. War wounds: lessons learned from Operation Iraqi Freedom. Plast Reconstr Surg. 2008;122(1):146-53.

2. Kumar AR. Standard wound coverage techniques for extremity war injury. J Am Acad Orthop Surg. 2006;14(10 Spec No):S62-5.

3. Kumar AR, Grewal NS, Chung TL, Bradley JP. Lessons from Operation Iraqi Freedom: successful subacute reconstruction of complex lower extremity battle injuries. Plast Reconstr Surg. 2009;123(1):218-29.

4. Klem C, Sniezek JC, Moore B, Davis MR, Coppit G, Schmalbach C. Microvascular reconstructive surgery in Operations Iraqi and Enduring Freedom: the US military experience performing free flaps in a combat zone. J Trauma Acute Care Surg. 2013;75 Suppl 2:S228-32.

5. Huh J, Stinner DJ, Burns TC, Hsu JR. Infectious complications and soft tissue injury contribute to late amputation after severe lower extremity trauma. J Trauma. 2011;71 Suppl 1:S47-51.

6. Theodorakopoulou E, Mason K, Ghanem A, Pafitanis G, Myers S, Iwuagwu F. Free-tissue transfer for the reconstruction of war-related extremity injuries: a systematic review of current practice. Eur Surg Res. 2015;55 Suppl 1:1-167.

7. Brown KV, Ramasamy A, McLeod J, Stapley S, Clasper JC. Predicting the need for early amputation in ballistic mangled extremity injuries. J Trauma. 2009:66 Suppl 4:593-7. discussion S7-8.

8. Spear M. Outcomes of lower extremity injuries sustained during Operation Iraqi Freedom and Operation Enduring Freedom. Plast Surg Nurs. 2009:29(3):155-7.

9. Doukas WC, Hayda RA, Frisch HM, Andersen RC, Mazurek MT, Ficke JR, et al. The Military Extremity Trauma Amputation/Limb Salvage (METALS) study: outcomes of amputation versus limb salvage following major lower-extremity trauma. J Bone Joint Surg Am. 2013;95(2):138-45.

10. Janis JE, Kwon RK, Attinger CE. The new reconstructive ladder: modifications to the traditional model. Plast Reconstr Surg. 2011;127:205-12S.

11. Valerio IL, Campbell P, Sabino J, Dearth $C L$, Fleming M. The use of urinary bladder matrix in the treatment of trauma and combat casualty wound care. Regen Med. 2015;10(5):611-22.

12. Tekin L, Zor F, Akarsu S, Tuncer SK, Ozturk S, Ozturk S. Quality of life and functionality of patients with heel reconstruction after landmine explosions. PM R. 2013;5(7):591-5.

13. Doucet JJ, Galarneau MR, Potenza BM, Bansal V, Lee JG, Schwartz AK, et al. Combat versus civilian open tibia fractures: the effect of blast mechanism on limb salvage. J Trauma. 2011;70(5):1241-7.

14. Godina M. Early microsurgical reconstruction of complex trauma of the extremities. Plast Reconst Surg. 1986;78(3):285-92.

15. Chattar-Cora D, Perez-Nieves R, McKinlay A, Kunasz M, Delaney R, Lyons R. Operation Iraqi Freedom: a report on a series of soldiers treated with free tissue transfer by a plastic surgery service. Ann Plast Surg. 2007;58(2):200-6.

16. Johansen K, Daines M, Howey T, Helfet D, Hansen Jr ST. Objective criteria accurately predict amputation following lower extremity trauma. J Trauma. 1990;30(5):568-72. discussion 572-73.

17. Casey K, Demers P, Deben S, Nelles ME, Weiss JS. Outcomes after long-term follow-up of combat-related extremity injuries in a multidisciplinary limb salvage clinic. Ann Vasc Surg. 2015;29(3):496-501.

18. Sabino J, Polfer E, Tintle S, Jessie E, Fleming M, Martin B, et al. A decade of conflict: flap coverage options and outcomes in traumatic war-related extremity reconstruction. Plast Reconstr Surg. 2015;135(3):895-902.

19. Burns TC, Stinner DJ, Possley DR, Mack AW, Eckel TT, Potter BK, et al. Does the zone of injury in combat-related Type III open tibia fractures preclude the use of local soft tissue coverage? J Orthop Trauma. 2010;24(11):697-703.

20. Casey K, Sabino J, Jessie E, Martin BD, Valerio I. Flap coverage outcomes following vascular injury and repair: chronicling a decade of severe warrelated extremity trauma. Plast Reconstr Surg. 2015;135(1):301-8.

21. Dickens JF, Kilcoyne KG, Kluk MW, Gordon WT, Shawen SB, Potter BK. Risk factors for infection and amputation following open, combat-related calcaneal fractures. J Bone Joint Surg Am. 2013;95(5):e24.

22. Harris AM, Althausen PL, Kellam J, Bosse MJ, Castillo R. Complications following limb-threatening lower extremity trauma. J Orthop Trauma. 2009;23(1):1-6.

23. Keeling JJ, Hsu JR, Shawen SB, Andersen RC. Strategies for managing massive defects of the foot in high-energy combat injuries of the lower extremity. Foot Ankle Clin. 2010;15(1):139-49.

24. Tintle SM, Keeling JJ, Shawen SB. Combat foot and ankle trauma. J Surg Orthop Adv. 2010;19(1):70-6.

25. Dini V, Romanelli M, Piaggesi A, Stefani A, Mosca F. Cutaneous tissue engineering and lower extremity wounds (part 2). Int J Low Extrem Wounds. 2006;5(1):27-34. 
26. Fleming ME, O'Daniel A, Bharmal H, Valerio I. Application of the orthoplastic reconstructive ladder to preserve lower extremity amputation length. Ann Plast Surg. 2014;73(2):183-9.

27. Helgeson MD, Potter BK, Evans KN, Shawen SB. Bioartificial dermal substitute: a preliminary report on its use for the management of complex combat-related soft tissue wounds. J Orthop Trauma. 2007;21(6):394-9.

28. Green 3rd JM, Sabino J, Fleming M, Valerio I. Intraoperative fluorescence angiography: a review of applications and outcomes in war-related trauma. Mil Med. 2015;180 Suppl 3:37-43.

29. Gustilo RB, Anderson JT. Prevention of infection in the treatment of one thousand and twenty-five open fractures of long bones: retrospective and prospective analyses. J Bone Joint Surg Am. 1976;58(4):453-8.

30. Melcer T, Sechriest VF, Walker J, Galarneau M. A comparison of health outcomes for combat amputee and limb salvage patients injured in Iraq and Afghanistan wars. J Trauma Acute Care Surg. 2013;75(2):S247-54.

31. Jeffery S. Challenges of treating military wounds. Nurs Stand. 2011;26(45):63-8.

32. Shores JT, Hiersche M, Gabriel A, Gupta S. Tendon coverage using an artificial skin substitute. J Plast Reconst Aesthet Surg. 2012;65(11):1544-50.

33. Valerio I, Sabino J, Thomas S, Tintle S, Fleming M, Shashikant M, et al. Multiple limbs salvaged using tissue transfers in the same casualty: a cohort comparison study chronicling a decade of war-injured patients. Plast Reconstr Surg. 2014; 134(2):333-8.

34. Saint-Cyr M, Wong C, Schaverien M, Mojallal A, Rohrich RJ. The perforasome theory: vascular anatomy and clinical implications. Plast Reconstr Surg. 2009; 124(5):1529-44.

35. Gordon WT, Stannard JP, Pasquina PF, Archer KR. Evolution of orthopaedic rehabilitation care. J Am Acad Orthop Surg. 2012;20 Suppl 1:S80-3.

\section{Submit your next manuscript to BioMed Central and we will help you at every step:}

- We accept pre-submission inquiries

- Our selector tool helps you to find the most relevant journal

- We provide round the clock customer support

- Convenient online submission

- Thorough peer review

- Inclusion in PubMed and all major indexing services

- Maximum visibility for your research

Submit your manuscript at www.biomedcentral.com/submit

C Biomed Central 